\title{
LOCAL RECURRENCE OF PHYLLODES TUMORS OF THE BREAST : A META-ANALYSIS
}

\author{
Ismail Abd Elhakim Mohammed*, Ahmed Gamal El Dein Othman*, \\ Mohammed Atef Mohammed Eltaib* and Ahmed Hamdy Fathy**
}

\begin{abstract}
:
Background: Phyllodes tumors (PTs) are uncommon

*General Surgery Department, Faculty of Medicine, Ain Shams University, Egypt

**Wady Elnatron

Specialized Hospital

Corresponding author

Ahmed Hamdy Fathy

Mobile: : (+2)01550484875

E.mail:

kingahmedking1@gmail.com

Received: $1 / 4 / 2021$

Accepted: $29 / 4 / 2021$

Online ISSN: $2735-3540$

fibroepithelial breast tumors that are capable of a diverse range of biologic behaviors. In their least aggressive form, phyllodes tumors behave like benign fibroadenomas, although with a propensity to recur locally following excision without wide margins.

Aim of Work: Our study focused on local recurrence and various risk factors of phyllodes tumors of the breast through a meta-analysis.

Patient and Methods: This Review involved case-control studies, case report studies, and retrospective case follow-up evaluating local recurrence (LR) rates of unilateral or bilateral phyllodes tumors. phyllodes tumors of breast and assessing various risk factors for $L R$.

Results: Forty-eight studies compared the LR risk between Benign / malignant. There was significant higher in Malignant VS Benign regarding rate of local recurrence p-value $<0.001$. Pooling of data from twenty-three showed significant difference in the LR risk between patients who underwent breast conserving surgery $(B C S)$ and those who had a mastectomy p-value 0.001 .

Conclusion: The risk of $L R$ was significantly increased from benign to borderline to malignant PTs, type of surgery, and surgical margin status may be risk factors for LR, while mitoses, tumor border, stromal cellularity, stromal atypia, stromal overgrowth, tumor necrosis had no difference regard increase risk for LR, Different management strategies could be considered for different PT grade.
\end{abstract}

Keywords: Phyllodes Tumors, Local Recurrence, breast tumors

\section{INTRODUCTION:}

Phyllodes tumors (PTs) are uncommon fibroepithelial breast tumors that are capable of a diverse range of biologic behaviors. In their least aggressive form, phyllodes tumors behave like benign fibroadenomas, although with a propensity to recur locally following excision without wide margins. At the other end of the spectrum, other phyllodes tumors can metastasize distantly, sometimes degenerating histologically into sarcomatous lesions that lack an epithelial component ${ }^{(\mathbf{1})}$.
The term "phyllodes," which means leaf-like, describes the typical papillary projections that are seen on pathologic examination. Although they were originally called "cystosarcoma phyllodes" by Johannes Müller in $1838^{(2)}$.

Phyllodes tumors only occasionally have cystic components and are not true sarcomas by either cellular origin or biologic behavior. The terminology has since evolved, with over 60 synonyms having been applied to this entity before the term 
"phyllodes tumors" was adopted by the World Health Organization ${ }^{(3)}$.

This study focused on local recurrence and various risk factors of phyllodes tumors of the breast through a meta-analysis.

\section{AIM OF THE WORK}

This review focuses on local recurrence and various risk factors of phyllodes tumors of the breast through a meta-analysis.

\section{PATIENTS AND METHODS}

The current study is a systematic review in which the following was conducted

- Type of studies: Review considered case-control studies, case report studies, and retrospective case follow-up evaluating local recurrence (LR) rates of phyllodes tumors of breast and assessing various risk factors for LR.

- Types of participants: This review considered all studies of unilateral or bilateral phyllodes tumors.

- Types of intervention: Interventions of interest included those related to which phyllodes tumors patients received surgical treatment.

\section{Types of outcome measures:}

The primary outcome of interest is reviewing Studies that included the LR rate with or without the following clinicopathologic factors were included: age, tumor size, surgery, surgical margin, tumor necrosis, stromal cellularity, stromal atypia, stromal overgrowth, mitoses, cellular pleomorphism, and tumor border. For risk factor analysis, only the studies reporting LR rates stratified by each risk factor were included. For age and tumor size, only the studies that used 40-year and 5-cm cutoff values, respectively, were included.
Search strategy for identification of studies:

Search strategy had been designed to include both manual and electronic data available. Electronic searches will involve searching databases of PubMed (from January 1995 till May 2018), EMbase, CINAHL and Cochrane database searching keywords and terms listed below:

- "Phyllodes tumor, breast, local recurrence"

- Also, full copies of articles of available medical journals and other published studies identified by the search, discussion with several investigators expert in the field and published case reports, considered to meet the inclusion criteria, based on their title, abstract and subject descriptors, had been obtained for data synthesis.

- Our review had been restricted to studies conducted in English language.

\section{Methods:}

\section{Locating and selecting studies:}

Abstracts of articles identified using the search strategy above had been viewed, and articles that appear to fulfill the inclusion criteria had been retrieved in full. Data on at least one of the outcome measures must be included in the study.

\section{RESULTS:}

Six studies compared the LR risk between two age subgroups (C 40 vs. $\backslash 40$ years). There was significant higher in $>40$ years VS $<40$ years regarding rate of local recurrence p-value 0.012 (Table 1).

Forty-eight studies compared the LR risk between Benign / malignant. There was significant higher in Malignant VS Benign regarding rate of local recurrence $p$-value $<0.001$ (Table 2). 


\section{Local Recurrence Of Phyllodes Tumors Of The Breast: A Meta-Analysis}

Pooling of data from twenty-three showed significant difference in the LR risk between patients who underwent breast conserving surgery (BCS) and those who had a mastectomy p-value 0.001 (Table 3).

A total of two studies assessed the association between the Tumor necrosis (positive VS negative) and LR, Collectively, a positive versus a negative Tumor necrosis no significantly difference p-value 0.879 (Figure 1).

A total of three studies assessed the association between the Stromal Cellularity (high VS low) and LR; there was no significantly difference p-value 0.465 (Figure 2).

A total of two studies assessed the association between the Stromal proliferation (yes VS no) and LR, there was no significantly difference p-value 0.573 (Figure 3).

A total of four studies assessed the association between the Mitoses $(\geq 5 \mathrm{VS}<5)$ and LR, there was no significantly difference p-value 0.005 (Figure 4).

Table 1: Comparison between studies regarding age

\begin{tabular}{|c|c|c|c|c|c|}
\hline Study & $>40$ yrs. & $<40 \mathrm{yrs}$. & Odd's ratio & $95 \% \mathrm{CI}$ & Random Weight (\%) \\
\hline Chaney. 2000 & $2 / 51$ & $2 / 50$ & 0.980 & $0.133-7.238$ & 9.42 \\
\hline Chen. 2005 & $13 / 73$ & $6 / 99$ & 3.358 & $1.211-9.317$ & 18.87 \\
\hline Kim S et al. 2013 & $10 / 104$ & $8 / 89$ & 1.077 & $0.406-2.859$ & 19.46 \\
\hline Wei. 2014 & $15 / 129$ & $16 / 63$ & 0.387 & $0.177-0.845$ & 22.09 \\
\hline Demian GA et al. 2016 & $5 / 35$ & $0 / 35$ & 12.803 & $0.680-241.043$ & 5.29 \\
\hline Zhou. 2018 & $28 / 233$ & $26 / 171$ & 0.762 & $0.429-1.353$ & 24.87 \\
\hline Total (fixed effects & $73 / 625$ & $58 / 507$ & 0.958 & 0.667 to 1.376 & 100.0 \\
\hline Total (random effects) & $73 / 625$ & $58 / 507$ & 1.103 & 0.523 to 2.326 & 100.0 \\
\hline Test for heterogeneity & & & & & \\
\hline $\mathrm{Q}$ & \multicolumn{5}{|c|}{14.6447} \\
\hline $\mathrm{DF}$ & \multicolumn{5}{|c|}{5} \\
\hline Significance level & \multicolumn{5}{|c|}{$0.012^{*}$} \\
\hline $\mathrm{I}^{2}$ (inconsistency) & \multicolumn{5}{|c|}{$65.86 \%$} \\
\hline $95 \%$ CI for $\mathrm{I}^{2}$ & \multicolumn{5}{|c|}{$18.24-85.74$} \\
\hline
\end{tabular}

Table 2: Comparison between studies regarding distribution as benign or malignant.

\begin{tabular}{|c|c|c|c|c|c|}
\hline Study & $\mathbf{1 / 3 0}$ & $\mathbf{3 / 5 9}$ & $\mathbf{0 . 6 4 4}$ & $\mathbf{0 . 0 6 4 1}$ to 6.467 & $\begin{array}{c}\text { Random Weight } \\
\text { (\%) }\end{array}$ \\
\hline Chaney. 2000 & $7 / 42$ & $2 / 52$ & 5.000 & $0.980-25.514$ & 1.21 \\
\hline Niezabitowski. 2001 & $3 / 9$ & $1 / 29$ & 14.000 & $1.234-158.851$ & 1.89 \\
\hline Sotheran. 2005 & $0 / 29$ & $19 / 131$ & 0.0978 & $0.00573-1.667$ & 1.12 \\
\hline Chen. 2005 & $6 / 25$ & $4 / 42$ & 3.000 & $0.755-11.923$ & 0.88 \\
\hline Renner. 2005 & $6 / 21$ & $3 / 31$ & 3.733 & $0.816-17.090$ & 2.25 \\
\hline Hassan. 2006 & $5 / 28$ & $3 / 62$ & 4.275 & $0.944-19.359$ & 2.04 \\
\hline Ben Hassouna. 2006 & $0 / 31$ & $20 / 138$ & 0.0918 & $0.00540-1.559$ & 2.06 \\
\hline Cheng. 2006 & $12 / 90$ & $23 / 203$ & 1.204 & $0.571-2.541$ & 0.88 \\
\hline Barrio. 2007 & $22 / 79$ & $31 / 284$ & 3.150 & $1.699-5.840$ & 3.42 \\
\hline Belkacemi. 2008 & $2 / 8$ & $3 / 34$ & 3.444 & $0.470-25.232$ & 3.67 \\
\hline Karim. 2009 & $5 / 12$ & $4 / 39$ & 6.250 & $1.333-29.302$ & 1.48 \\
\hline Jung. 2010 & $0 / 6$ & $7 / 114$ & 1.103 & $0.0566-21.496$ & 2.01 \\
\hline Guillot. 2011 & $6 / 20$ & $15 / 90$ & 2.143 & $0.709-6.473$ & 0.82 \\
\hline Tsang. 2012 & $10 / 40$ & $12 / 82$ & 1.944 & $0.758-4.987$ & 2.72 \\
\hline Jang. 2012 & $1 / 1$ & $5 / 50$ & 24.818 & $0.897-686.973$ & 3.03 \\
\hline Ga-Eon Kim. 2012 & $9 / 50$ & $48 / 399$ & 1.605 & $0.734-3.509$ & 0.68 \\
\hline
\end{tabular}


Ismail Abd Elhakim Mohammed, et al.,

\begin{tabular}{|c|c|c|c|c|c|}
\hline Tan. 2012 & $26 / 49$ & $16 / 77$ & 4.310 & $1.964-9.459$ & 3.35 \\
\hline Ramakant. 2013 & $2 / 17$ & $7 / 120$ & 2.152 & $0.409-11.334$ & 3.34 \\
\hline Ho. 2013 & $8 / 62$ & $3 / 68$ & 3.210 & $0.811-12.697$ & 1.85 \\
\hline Spitaleri. 2013 & $7 / 15$ & $5 / 145$ & 24.500 & $6.346-94.586$ & 2.26 \\
\hline Kim S et al. 2013 & $6 / 42$ & $2 / 42$ & 3.333 & $0.632-17.574$ & 2.30 \\
\hline Sawalhi S et al. 2013 & $2 / 32$ & $16 / 106$ & 0.375 & $0.0814-1.727$ & 1.85 \\
\hline Huang. 2014 & $12 / 49$ & $9 / 80$ & 2.559 & $0.988-6.624$ & 2.03 \\
\hline Wei. 2014 & $9 / 47$ & $17 / 125$ & 1.505 & $0.619-3.659$ & 3.01 \\
\hline Wang Hui. 2014 & $21 / 38$ & $15 / 95$ & 6.588 & $2.832-15.328$ & 3.14 \\
\hline Narayanakar. 2015 & $3 / 11$ & $11 / 75$ & 2.182 & $0.500-9.519$ & 3.22 \\
\hline Xiao. 2015 & $2 / 11$ & $3 / 57$ & 4.000 & $0.584-27.376$ & 2.11 \\
\hline Ng. 2015 & $5 / 33$ & $8 / 191$ & 4.085 & $1.248-13.374$ & 1.55 \\
\hline Yom. 2015 & $3 / 16$ & $4 / 105$ & 5.827 & $1.171-28.992$ & 2.58 \\
\hline Akrami. 2015 & $1 / 32$ & $12 / 179$ & 0.449 & $0.0563-3.578$ & 1.93 \\
\hline Ruvalcaba-Limon. 2016 & $7 / 16$ & $5 / 153$ & 23.022 & $6.087-87.081$ & 1.40 \\
\hline Kim. 2016 & $2 / 9$ & $7 / 40$ & 1.347 & $0.229-7.912$ & 2.33 \\
\hline Bellezza. 2016 & $8 / 91$ & $22 / 354$ & 1.455 & $0.625-3.383$ & 1.72 \\
\hline $\begin{array}{c}\text { Borhani-Khomani K et } \\
\text { al. } 2016\end{array}$ & $2 / 35$ & $1 / 35$ & 2.061 & $0.178-23.827$ & 3.23 \\
\hline Demian GA et al. 2016 & $0 / 13$ & $3 / 81$ & 0.831 & $0.0406-17.005$ & 1.11 \\
\hline Tremblay-LeMay. 2017 & $3 / 11$ & 9/30 & 0.875 & $0.188-4.080$ & 0.80 \\
\hline Matos. 2017 & $3 / 16$ & $5 / 55$ & 2.308 & $0.487-10.938$ & 2.01 \\
\hline Varghese. 2017 & $3 / 64$ & $21 / 281$ & 0.609 & $0.176-2.107$ & 1.99 \\
\hline Co M et al. 2017 & $7 / 49$ & $5 / 81$ & 2.533 & $0.757-8.478$ & 2.48 \\
\hline $\begin{array}{c}\text { Rodrigues MF et al. } \\
2017\end{array}$ & $22 / 52$ & $6 / 168$ & 19.800 & $7.408-52.925$ & 2.54 \\
\hline Zhou. 2018 & $7 / 49$ & $5 / 81$ & 2.533 & $0.757-8.478$ & 2.95 \\
\hline Rodrigues. 2018 & $9 / 53$ & $0 / 9$ & 4.056 & $0.217-75.877$ & 2.54 \\
\hline Ganesh. 2018 & $2 / 17$ & $9 / 196$ & 2.770 & $0.548-13.998$ & 0.84 \\
\hline Chng. 2018 & $0 / 21$ & $10 / 45$ & 0.0786 & $0.00438-1.411$ & 1.90 \\
\hline Slodknowska. 2018 & $3 / 64$ & $21 / 281$ & 0.609 & $0.176-2.107$ & 0.86 \\
\hline Co. 2018 & $6 / 10$ & $16 / 33$ & 1.594 & $0.379-6.711$ & 2.48 \\
\hline Wang K. 2018 & $3 / 8$ & $13 / 95$ & 3.785 & $0.806-17.766$ & 2.16 \\
\hline Wada A et al. 2018 & $289 / 1553$ & $489 / 5322$ & 2.214 & $1.872-2.617$ & 2.00 \\
\hline Total (fixed effects) & $289 / 1553$ & 489/5322 & 2.482 & $1.842-3.344$ & 100.00 \\
\hline Total (random effects) & $1 / 30$ & 3/59 & 0.644 & $0.0641-6.467$ & 100.00 \\
\hline Test for heterogeneity & \multirow{2}{*}{\multicolumn{5}{|c|}{105.2413}} \\
\hline $\mathbf{Q}$ & & & & & \\
\hline DF & \multicolumn{5}{|c|}{47} \\
\hline Significance level & \multicolumn{5}{|c|}{$<0.001^{*}$} \\
\hline$I^{2}$ (inconsistency) & \multicolumn{5}{|c|}{$55.34 \%$} \\
\hline $95 \%$ CI for $\mathrm{I}^{2}$ & \multicolumn{5}{|c|}{$38.20-67.73$} \\
\hline
\end{tabular}

Table 3: Comparison between studies regarding Mastectomy/Breast-conserving surgery.

\begin{tabular}{|c|c|c|c|c|c|}
\hline Study & Mastectomy & $\begin{array}{c}\text { Breast-con. } \\
\text { surgery }\end{array}$ & Odds ratio & $95 \%$ CI & $\begin{array}{c}\text { Random } \\
\text { Weight }(\%)\end{array}$ \\
\hline Chaney. 2000 & $2 / 54$ & $2 / 47$ & 0.865 & $0.117-6.396$ & 2.80 \\
\hline Asoglu. 2004 & $8 / 28$ & $8 / 22$ & 0.700 & $0.212-2.311$ & 5.03 \\
\hline Chen. 2005 & $0 / 46$ & $19 / 126$ & 0.0593 & $0.0035-1.003$ & 1.66 \\
\hline Sotheran. 2005 & $0 / 8$ & $7 / 42$ & 0.278 & $0.0144-5.368$ & 1.54 \\
\hline Renner. 2005 & $4 / 15$ & $11 / 55$ & 1.455 & $0.388-5.453$ & 4.58 \\
\hline Tan. 2005 & $4 / 23$ & $38 / 311$ & 1.512 & $0.488-4.684$ & 5.27 \\
\hline Hassan. 2006 & $2 / 33$ & $14 / 46$ & 0.147 & $0.031-0.703$ & 3.83 \\
\hline Ben Hassouna. 2006 & $10 / 24$ & $3 / 82$ & 18.810 & $4.593-77.033$ & 4.29 \\
\hline Barrio. 2007 & $4 / 48$ & $31 / 242$ & 0.619 & $0.208-1.842$ & 5.43 \\
\hline
\end{tabular}


Local Recurrence Of Phyllodes Tumors Of The Breast: A Meta-Analysis

\begin{tabular}{|c|c|c|c|c|c|}
\hline Jung. 2010 & $2 / 5$ & $9 / 62$ & 3.926 & $0.573-26.878$ & 2.95 \\
\hline Jang. 2012 & $3 / 13$ & $28 / 148$ & 1.286 & $0.332-4.981$ & 4.47 \\
\hline Spitaleri. 2013 & $6 / 35$ & $14 / 137$ & 1.818 & $0.643-5.135$ & 5.63 \\
\hline Ramakant. 2013 & $19 / 56$ & $33 / 94$ & 0.949 & $0.473-1.905$ & 7.09 \\
\hline Kim S et al. 2013 & $3 / 11$ & $20 / 182$ & 3.038 & $0.745-12.390$ & 4.30 \\
\hline Xiao. 2015 & $0 / 9$ & $21 / 118$ & 0.239 & $0.0134-4.260$ & 1.61 \\
\hline Narayanakar. 2015 & $13 / 63$ & $35 / 99$ & 0.475 & $0.228-0.993$ & 6.92 \\
\hline Yom. 2015 & $1 / 14$ & $19 / 271$ & 1.020 & $0.127-8.222$ & 2.64 \\
\hline Bellezza. 2016 & $0 / 9$ & $12 / 53$ & 0.175 & $0.009-3.218$ & 1.58 \\
\hline $\begin{array}{c}\text { Demian GA et al. } \\
2016\end{array}$ & $3 / 35$ & $2 / 35$ & 1.547 & $0.242-9.879$ & 3.10 \\
\hline Matos. 2017 & $3 / 12$ & $14 / 40$ & 0.619 & $0.144-2.664$ & 4.13 \\
\hline Zhou. 2018 & $4 / 26$ & $50 / 378$ & 1.193 & $0.395-3.605$ & 5.36 \\
\hline Choi N et al. 2018 & 9/97 & $51 / 265$ & 0.429 & $0.203-0.909$ & 6.86 \\
\hline Efared B. 2018 & $1 / 22$ & $2 / 84$ & 1.952 & $0.169-22.577$ & 2.09 \\
\hline Total (fixed effects) & $110 / 783$ & $494 / 3204$ & 0.746 & $0.590-0.943$ & 100.00 \\
\hline $\begin{array}{l}\text { Total (random } \\
\text { effects) }\end{array}$ & $110 / 783$ & $494 / 3204$ & 0.910 & $0.608-1.362$ & 100.00 \\
\hline Test for heterogeneity & & & & & \\
\hline $\mathrm{Q}$ & \multicolumn{5}{|c|}{50.1928} \\
\hline $\mathrm{DF}$ & \multicolumn{5}{|c|}{23} \\
\hline Significance level & \multicolumn{5}{|c|}{$0.001^{*}$} \\
\hline $\mathrm{I}^{2}$ (inconsistency) & \multicolumn{5}{|c|}{$54.18 \%$} \\
\hline $95 \%{\mathrm{CI} \text { for } \mathrm{I}^{2}}^{2}$ & \multicolumn{5}{|c|}{$27.27-71.13$} \\
\hline
\end{tabular}

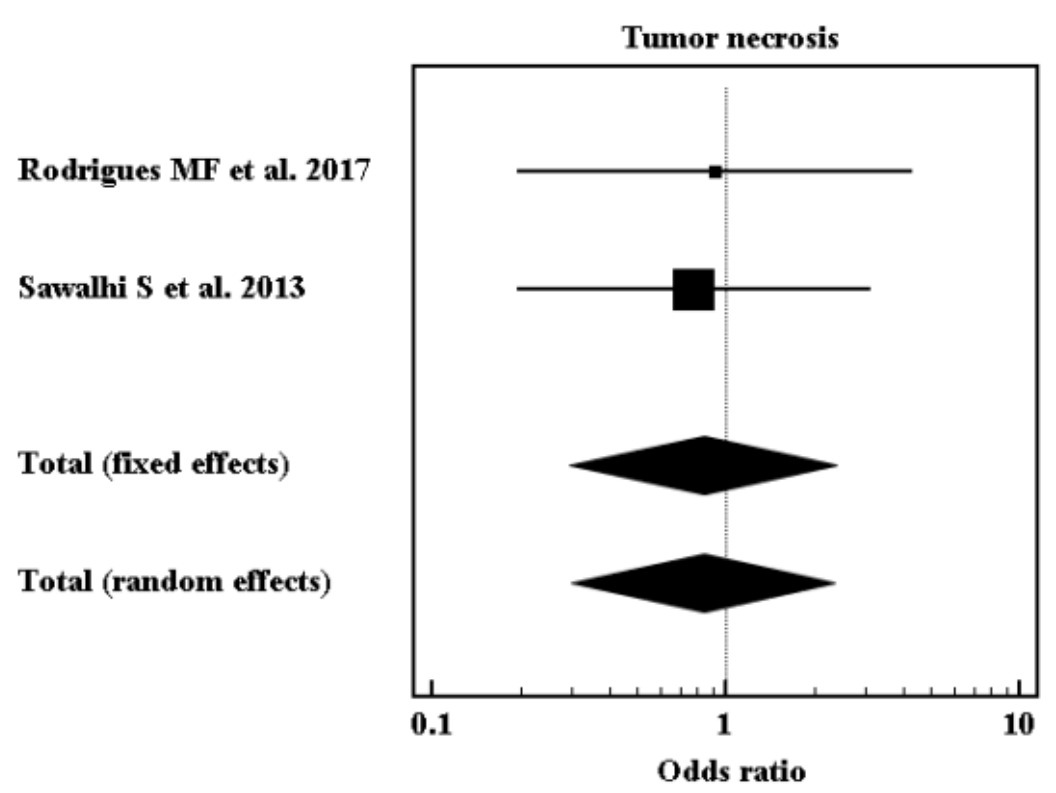

Diagram (1): Forest plot for tumor necrosis. 


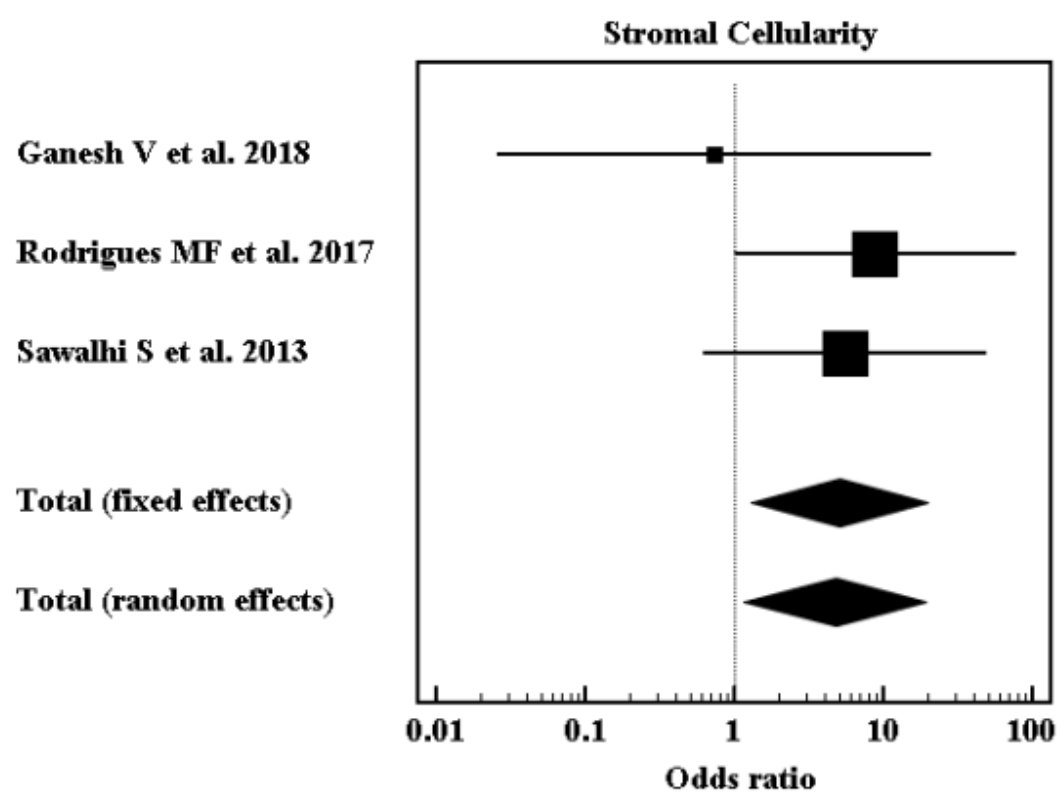

Diagram (2): Forest plot for Stromal Cellularity (high VS low).

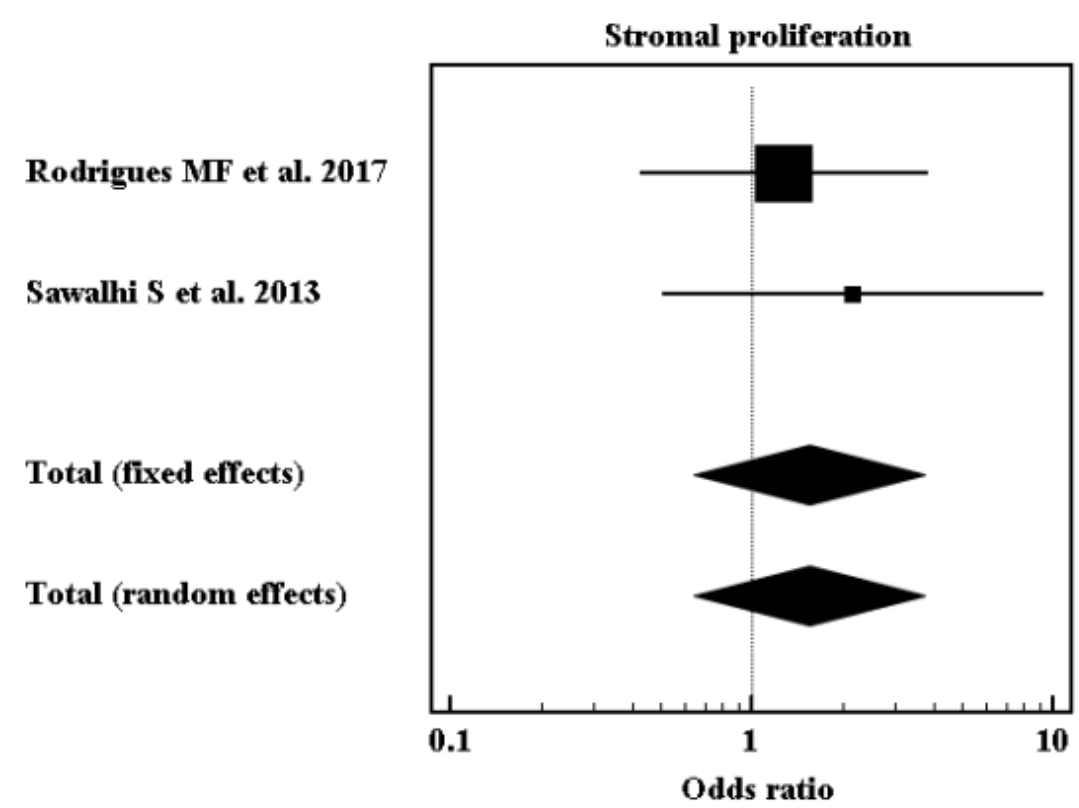

Diagram (3): Forest plot for Stromal proliferation. 


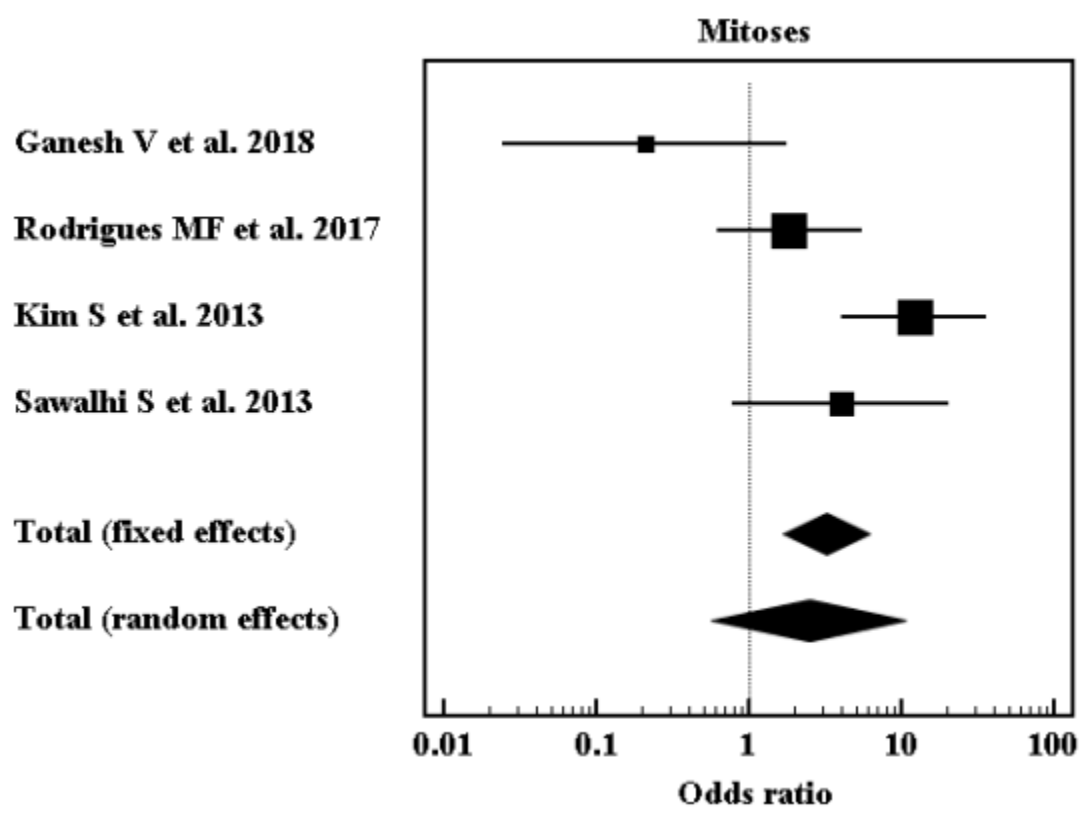

Diagram (4): Forest plot for mitoses.

\section{DISCUSSION:}

To date, no large-scale prospective studies of PTs have been conducted due to their low incidence. Therefore, the existing guidelines for PTs are based on retrospective studies, and data are limited. We performed a systematic review and meta-analysis to evaluate LR rates comprehensively for each PT grade and to investigate the related risk factors.

In the present study, forty-seven studies compared the LR risk between Benign/Borderline. There was significant higher in Borderline vs benign regarding rate of local recurrence p-value $<0.001$. And forty-seven studies compared the LR risk between benign / malignant. There was significant higher in malignant vs Benign regarding rate of local recurrence $p$-value $<0.001$.

In ${ }^{(4)}$ study, the LR rates increased from benign (8\%; range, 6-9\%) to borderline (13\%; range, $11-16 \%)$ to malignant $(18 \%$; range, $14-21 \%$ ) PTs. The lower limit of the pooled OR of the malignant versus the borderline PTs was close to 1.00 (OR 1.28;
95\% CI 1.05-1.55). Additionally, the 95\% CIs of the pooled LR rates for the borderline and malignant PTs overlapped, indicating that some borderline cases may recur at a risk as high as for malignant PTs.

These findings suggested that borderline PTs may deserve the same attention as malignant PTs during surgical decision making. Notably, some benign PTs recurred as borderline and malignant PTs ${ }^{\mathbf{5} \& 6)}$.

Xiao, M. et al., ${ }^{(7)}$ reported that of the 127 women, the pathologic diagnoses were benign, borderline, and malignant phyllodes tumors in $75(59.1 \%), 41$ (32.3\%), and 11 $(8.7 \%)$ patients, The benign, borderline, and malignant tumor recurrence rates were $14.7 \%, 17.1 \%$, and $27.3 \%$, respectively.

The primary treatment for borderline and malignant phyllodes tumors is wide local excision (margins $\geq 1 \mathrm{~cm}$ ), in the context of either breast-conserving surgery (BCS) or total mastectomy (TM). Local recurrence (LR) occurs in $10-65 \%$ of the patients and distant recurrence rates range between 5 and $40 \%$ postoperatively ${ }^{(8)}$. Due to the high risk of LR following surgical 
intervention alone, the addition of adjuvant radiotherapy (RT) to the treatment of borderline and malignant phyllodes tumors has been previously investigated; however, the conclusions have been inconsistent. Furthermore, an observational study reported a trend toward increased utilization of RT, despite its uncertain effect on outcome. The current guidelines only recommend consideration of $\mathrm{RT}$ for malignant phyllodes in the setting of LR (level 2B evidence) ${ }^{(9)}$.

Pooling of data in our systematic review, from twenty three showed significant difference in the LR risk between patients who underwent breast conserving surgery (BCS) and those who had a mastectomy p-value 0.001 .

Gnerlich, J. L., et al., ${ }^{(9)}$ reported a significant increase in RT use for BCS as well as TM patients, despite its uncertain efficacy. Therefore, we performed this systematic review and meta-analysis to integrate the results from recent studies that investigated the effect of adjuvant RT on borderline and malignant phyllodes tumors.

Bhargav, P. R. K., et al., ${ }^{(10)}$ believed that regardless of how the histological grade, wide local excision should be the first choice of surgical approach, but all patients with disease recurrence were required to undergo mastectomy.

Associations between frequently used pathologic parameters and the risk of LR also were scrutinized in the study of (4) where The pooled results showed that an increased risk of LR mitoses was significantly associated with 10/10 HPF or higher (OR 2.89; 95\% CI 1.40-5.97), an infiltrating versus a pushing border (OR 2.79 ; 95\% CI 1.43-5.46), moderate/severe versus mild stromal cellularity (OR 2.63; 95\% CI 1.58-4.39), severe versus mild/absent stromal atypia (OR 2.32, 95\% CI 1.08-4.96), severe versus mild/absent stromal overgrowth (OR 2.04, 95\% CI 1.03-
4.04), and positive versus negative tumor necrosis (OR 2.00; 95\% CI 1.17-3.40).

Korpanty, G. et al., ${ }^{(11)}$ noted that mitosis, cellular atypia, stromal overgrowth, and tumor necrosis were predictive of disease-free survival on univariate analysis, but the significance disappeared on multivariate analysis for all types of phyllodes tumors.

\section{Conclusion:}

The risk of LR was significantly increased from benign to borderline to malignant PTs, type of surgery, and surgical margin status may be risk factors for LR, while mitoses, tumor border, stromal cellularity, stromal atypia, stromal overgrowth, tumor necrosis had no difference regard increase risk for LR, Different management strategies could be considered for different PT grade. Local recurrence may occur in benign, borderline, and malignant phyllodes tumors. Therefore, early and periodic sonographic examinations are necessary after phyllodes tumor surgery to detect recurrence or metastases.

\section{REFERENCES:}

1. Calhoun K, Allison KH, Kim JN, et al. (2014): Phyllodes tumors. In: Diseases of the breast, Harris J, Lippman ME, Morrow M, Osborne KC. (Eds), Lippincott Williams and Wilkins.

2. Grau, A. M., Chakravarthy, A. B., \& Chugh, R. (2014): Phyllodes tumors of the breast. UpToDate. Available from: http://www. uptodate. com/home/index. html. Accessed September, 24.

3. Tavassoli FA and Devilee P. (2013): genetics of tumors of the breast and female genital organs. In: World Health Organization Classification of Tumors, IARC Press, Lyons. P.99.

4. Huang, C. C., Liu, T. P., Cheng, S. P., \& Chang, Y. C. (2014): Surgical treatment of phyllodes tumor of the breast with the 
trend. Journal of Experimental \& Clinical Medicine, 6(5), 161-165.

5. Barth, R. J. (2013): Margin negative, breast conserving resection: adequate for benign phyllodes tumors, but inadequate therapy for borderline and malignant phyllodes tumors. Breast cancer research and treatment, 142(2), 463-464.

6. Lu, Y., Chen, Y., Zhu, L., Cartwright, P., Song, E., Jacobs, L., et al. (2019): Local recurrence of benign, borderline, and malignant phyllodes tumors of the breast: a systematic review and metaanalysis. Annals of surgical oncology, 26 (5), 1263-1275.

7. Moutte, A., Chopin, N., Faure, C., Beurrier, F., Ho Quoc, C., Guinaudeau, F., et al. (2016): Surgical management of benign and borderline phyllodes tumors of the breast. The breast journal, 22(5), 547552 .
8. Xiao, M., Zhu, Q., Jiang, Y., Li, J., Wang, H., Zhang, J., et al. (2015): Local recurrent phyllodes tumors of the breast: clinical and sonographic features. Journal of Ultrasound in Medicine, 34(9), 16311638.

9. Gnerlich, J. L., Williams, R. T., Yao, K., Jaskowiak, N., \& Kulkarni, S. A. (2014): Utilization of radiotherapy for malignant phyllodes tumors: analysis of the National Cancer Data Base, 1998-2009. Annals of surgical oncology, 21(4), 1222-1230.

10. Bhargav, P. R. K., Mishra, A., Agarwal, G., Agarwal, A., Verma, A. K., \& Mishra, S. K. (2009): Phyllodes tumour of the breast: clinicopathological analysis of recurrent vs. non-recurrent cases. Asian journal of surgery, 32(4), 224-228.

11. Korpanty, G., Power, D. G., Murphy, C., Kell, M., \& McCaffrey, J. (2011): Phyllodes tumor of the breast. Medical Oncology, 28(1), 62-64.

\section{التكرار الموضعى للأورام الثيية فى الثي: دراسة مرجعية}

$$
\begin{aligned}
& \text { المقدمة: عادة ما تكون أور ام الثديية من الثذي كبيرة الحجم و سريعة النمو تمثل ما يصل إلى ا ـ ٪ من جميع الأورام }
\end{aligned}
$$

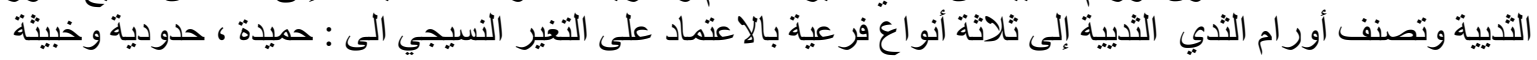

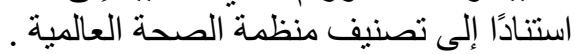

$$
\begin{aligned}
& \text { الهذف من الدراسة: دراسة مرجعية لتقيم حدوث التكرار الموضعي للأور ام الثديية بالثدي مع مناقثة بعض العوامل }
\end{aligned}
$$

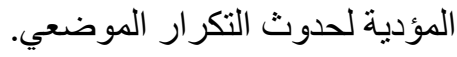

$$
\begin{aligned}
& \text { الحالات : الدراسات المقارنة والدراسات المرجعية للورم من الثذي او الثديين مع دراسة عوامل مختلفه لارتجاع } \\
& \text { الورم }
\end{aligned}
$$

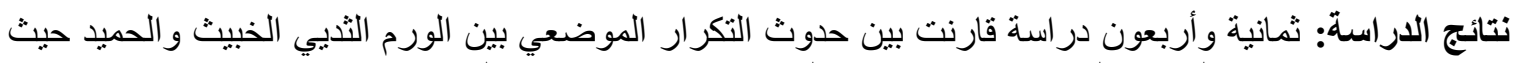

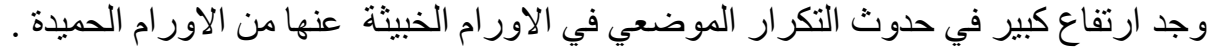

$$
\begin{aligned}
& \text { الاستنتاج: حدوث تكرار موضعي للورم يزداد في الاورام الخبيثة عن الحدودية عن الحميدة , طريقة الجراحة }
\end{aligned}
$$

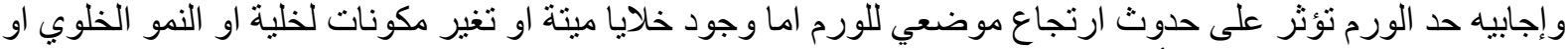

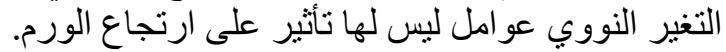

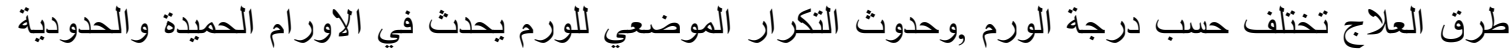

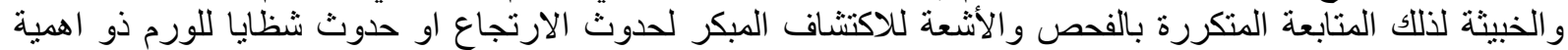

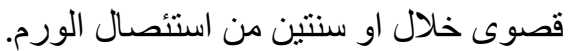

